\title{
DRAMATIZACIÓN COMO RECURSO DIDÁCTICO Y EXPRESIÓN ORAL DEL INGLÉS EN ESTUDIANTES DE EDUCACIÓN SECUNDARIA
}

\author{
DRAMATIZATION AS A DIDACTIC RESOURCE AND ORAL \\ EXPRESSION OF ENGLISH IN SECONDARY EDUCATION STUDENTS \\ (iD) Wilver Hermoza Palomino ${ }^{{ }^{*}}$ \\ wilverhermoza@hotmail.com \\ ${ }^{1}$ Universidad Nacional de San Cristóbal de Huamanga, Ayacucho, Perú
}

*Correspondencia: Wilver Hermoza Palomino. Email: wilverhermoza@hotmail.com

Recibido: 16.12.20 | Aprobado: 11.02.21

\section{RESUMEN}

La investigación tuvo como objetivo determinar el grado de influencia de la dramatización como recurso didáctico en el nivel de la expresión oral del inglés. El tipo de investigación fue empírico con diseño cuasiexperimental, en el que participaron 35 estudiantes del primer año de educación secundaria de la IE. "Señor de Atoccasa"Huancavelica, divididos en dos grupos, control (18) y experimental (17). Los instrumentos fueron una ficha de observación estructurada y la lista de chequeo, aplicados en 30 sesiones de clases. Los resultados señalan que los estudiantes del grupo experimental obtuvieron mejores logros en la evaluación del nivel de expresión oral del idioma inglés, mediado por la implementación rigurosa de los procedimientos de la dramatización.

Palabras clave: Dramatización, recurso didáctico, expresión oral del inglés.

\section{ABSTRACT}

The objective of the research was to determine the degree of influence of dramatization as a didactic resource on the level of oral expression of English. The type of research was empirical with a quasi-experimental design, in which 35 students of the first year of secondary education of EI participated. "Señor de Atoccasa" -Huancavelica, divided into two groups, control (18) and experimental (17). The instruments were a structured observation sheet and the checklist, applied in 30 class sessions. The results indicate that the students of the experimental group obtained better achievements in the evaluation of the level of oral expression of the English language, mediated by the rigorous implementation of the dramatization procedures.

Keywords: Dramatisation, didactic resource, English oral expression. 


\section{INTRODUCCIÓN}

En un entorno socio cultural donde el habla general y más usada es el quechua, las actividades oficiales y los procesos educativos se desarrollan en español, enseñar y aprender una nueva lengua es una tarea compleja, pero necesaria, pues en el mundo actual el idioma de uso común es el inglés (Chávez y otros, 2017). Por ello, a pesar de las dificultades que encierra, incluso el aprendizaje del español; la enseñanza del inglés es crucial para darle calidad a cualquier proceso educativo. Al respecto, Pérez (2015) señala que:

A nivel nacional (Colombia) no es desconocida la importancia que los niños y jóvenes adquieran una segunda lengua, porque ello les facilitará desenvolverse mejor en muchos campos, donde el inglés ha dejado de ser un complemento en la formación y pasa a ser una exigencia personal, profesional y sobre todo académica. (p. 3)

En efecto, en el campo laboral las empresas han convertido al inglés en un requerimiento para acceder a un empleo (España, 2009). El uso del internet contribuye en gran medida al fenómeno de la globalización debido a su avance vertiginoso; sin duda éste hecho provocó que el 52,3\% del contenido de la información digital este escrita en inglés (World Stats y W3Tech, 2017)

Contextualizando la información diremos que esta realidad problemática no solo es evidente, en la provincia de Churcampa, Huancavelica, sino en toda la región andina, y debe ser afrontada con políticas públicas eficientes. Sobre el particular, la UNESCO (2003) refiere una "educación plurilingüe" sustentada en el uso de por lo menos tres lenguas en la educación: la lengua materna, una lengua regional o nacional y una lengua internacional. Reconoce la importancia de recuperar y fortalecer la lengua materna, como complemento de la lengua general, y la necesidad de la enseñanza de un idioma de mayor uso a nivel universal, para que tenga las oportunidades que ofrece el mundo actual. Ante este conglomerado de realidades y demandas del futuro de los estudiantes, en la investigación se optó por utilizar otra forma de lenguaje para la enseñanza de la oralidad del inglés mediante la dramatización.

\section{MANIFESTACIONES DE UNA SITUACIÓN PROBLEMÁTICA}

Las diferentes evaluaciones censales, según los estudios de Llanos (2018) indican que el nivel de aprendizaje del inglés es muy bajo. El sistema educativo simplemente no está generando estudiantes con los niveles necesarios de dominio fundamentalmente de la expresión oral. Las escuelas con frecuencia son incapaces de impartir las clases de inglés necesarias, y si lo hacen son de baja calidad. Las oportunidades de aprendizaje fuera del sistema educativo, aunque cada vez están más disponibles, no logran compensar las deficiencias. En el caso del Perú, el 49,53\% de estudiantes se ubican en el nivel bajo del dominio del inglés (English First, 2016). El plan educativo del Perú para implementar un programa nacional de la enseñanza del inglés, considera el dominio de este idioma como una forma de atraer la inversión extranjera, lo que podría ayudar a aumentar la productividad y competitividad (Ministerio de Educación de Perú, 2016); sin embargo, los sistemas educativos en América Latina tienen un desempeño menor en enseñanza del inglés que los países de Asia y Europa (English First, 2016). 
En el Perú, consideran cinco horas de inglés a la semana, cubriendo tan solo, el $28 \%$ de la población de estudiantes. El 2017, cuatrocientas escuelas más se convirtieron en planteles de jornada completa, para alcanzar un total de 2004 escuelas; lo que indica que más estudiantes tendrán acceso a las clases de inglés (Instituto de Integración, 2016). Se esperaba que el 2017, en más de 5000 escuelas secundarias de medio tiempo se impartirían clases de inglés tres horas a la semana (MINEDU, 2016). Esto implica que el $75 \%$ de las escuelas secundarias públicas del Perú estuvieron impartiendo clases de inglés hacia el final de ese año.

Según Chiclote (2005) "las instituciones educativas de la sierra del Perú, en su totalidad no cuentan con un laboratorio especializado para fortalecer las capacidades y habilidades relacionadas con la expresión oral en el idioma inglés" (p.12). Además, se percibe esta dificultad particularmente en los primeros años de la educación secundaria, quiere decir los estudiantes no pronuncian con precisión y corrección los vocablos, tampoco trasmiten de manera coherente sus ideas (Ninamango, 2008).

Asimismo, a nivel regional según el reporte del informe sobre el concurso remitido por la Dirección Regional de Educación Huancavelica (DREH, 2017) los estudiantes presentan mayor deficiencia en la expresión oral en el idioma inglés, situación que se debe tener en cuenta para una formación integral, porque limita la interrelación y desenvolvimiento adecuado en el ámbito académico y social, más aún cuando se trata de un mundo globalizado, no obstante, los docentes le dan poca importancia, circunscribiéndose solo a la gramática, pero no tienen oportunidades para hacer uso permanente de esta lengua extranjera en sus procesos de comunicación.

El diagnóstico realizado (Fuente: Guía de observación) refleja que los estudiantes del primer año de educación secundaria presentan dificultades para expresar oralmente sus ideas en el idioma inglés, "La pronunciación es un aspecto esencial para una comunicación eficiente. Si el estudiante no produce los sonidos de forma inteligible no será capaz de comunicarse, lo que traerá como consecuencia interferencias en la comunicación” (Reyes y Echevarría, 2016, p.1). En efecto, no se trata solo de escribir, sino pronunciar y lograr comunicarse; se torna complejo que estudiantes bilingües (quechua hablantes) muestren visible temor para expresarse oralmente en inglés, a ello se suma la falta de interés que se refleja en una pronunciación poco comprensible escaso de pausas, articulación débil, además se percibe la falta de inflexiones tonales y matices semánticos; por ello, la intensidad de la expresión presenta un volumen inapropiado.

Otro aspecto, es la fluidez durante la comunicación; no muestran prolongación discursiva, sus ideas son limitadas y recortadas, lo único que repiten y han aprendido de memoria es preguntar cuál es tu nombre, repetir los colores, el abecedario y algunos nombres de objetos, animales y frutas, cada término responde a una pronunciación aislada del texto. En síntesis, el problema fundamental, que presentan es la falta de necesidad, por el aprendizaje de una lengua extranjera. Por ello, la riqueza del vocabulario es restringida, sus entornos familiares y sociales no consideran importante y las metodologías de trabajo no son pertinentes, en este contexto se propone una novedosa forma de trabajo académico mediada por la dramatización. 
Respecto a la categoría dramatización, Cervera (1992) manifiesta que es la acción, convencionalmente repetida. Y para esta representación hay que servirse de los recursos expresivos que la vida emplea para producir la acción, es decir, la expresión lingüística, corporal, plástica y la expresión rítmico-musical. Para Fuégel (2000) es encarnar personajes y producir escenas. Es una forma de expresión individual y social, poco aprovechado por los profesores. Quiere decir, se percibe como una interacción de las relaciones personales. "Dar a los estudiantes una experiencia (dryrun) de utilizar el lenguaje para una genuina comunicación y utilidad en la vida real; y para generar una necesidad de hablar" (Desialova, 2009, p.34). En efecto, este proceso provocará en el estudiante el interés de utilizar en su comunicación cotidiana los contenidos aprendidos en la clase.

Respecto al valor de la dramatización, Munther citado en Arksey y O’Maley (2005) postula que ésta integra las habilidades de la lengua de manera natural, sus aspectos verbales y no verbales, uniendo mente y cuerpo, y restaura el balance entre los aspectos físicos e intelectuales, porque utiliza ambos dominios afectivos y cognitivos, así devuelve la importancia del sentimiento y del pensamiento. Contextualiza el lenguaje, trae la interacción del aula a la vida diaria a través de un intenso enfoque de significado, poniendo énfasis en el aprendizaje y las aportaciones multisensoriales, ayuda al estudiante a fortalecer su capacidad y ampliar su alcance.

Por los argumentos señalados, las metodologías activas, como es el caso de la dramatización ofrecen oportunidades, para atender las diferencias de los estudiantes a partir de un proceso de toma de conciencia de sí mismo, fortalece la autoestima y la confianza para mantenerse motivado. Fomenta un estilo flexible, exploratorio del aprendizaje, donde la creatividad y la imaginación se integran; a su vez, promueve la toma de riesgos, que es un elemento esencial para un aprendizaje afectivo del idioma, porque genera un efecto positivo en la dinámica y la atmósfera del aula, facilita la difusión del conocimiento y su asimilación amigable; con respecto a los recursos, todo lo que se necesita son estudiantes comprometidos y predispuestos a aprender.

Intervención: la dramatización como un recurso didáctico. En esta parte de la investigación fue necesario desarrollar los fundamentos que sustentan la propuesta. La pedagogía, nos permite entender, que el desarrollo de los conceptos espontáneos del estudiante procede de modo ascendente y los conceptos científicos en forma descendente, hacia un nivel más elemental y concreto. El objeto es la realidad en la que el estudiante contextualiza lo que aprende, esa contextualización mediada por la familia y la sociedad, puede ser dramatizada, a partir de los impactos que puedan tener en el entorno los conocimientos adquiridos, si el idioma no es el propio, el proceso es más complejo (Vygotsky, 1995).

Los conceptos científicos describen el desarrollo mental del estudiante como análogo y complementario al aprendizaje. Al hacerlo en un idioma extranjero, consciente y deliberado, implica que la relación del aprendizaje de una lengua extranjera es parte de lo que ya conoce, porque articula con sus saberes estos dos procesos, al parecer distintos, pero se unifican al dramatizar y se genera un aprendizaje significativo. 
Desde una comprensión filosófica, Habermas (2007) señala la necesidad de vincular la teoría y la práctica, para lograr aprendizajes, este proceso se fundamenta en dinámicas comunicacionales. Adquieren su mayor concretización en los procesos de enseñanza; las obras creadas por los propios estudiantes, al ser expresadas en una lengua extranjera, adquieren mayor importancia. Todo aprendizaje tiene tres pilares: un interés técnico, donde el alumno relaciona y utiliza para cambiar su realidad; otro práctico, que le da sentido a las cosas, explicándolas a partir del nuevo conocimiento, y una vez familiarizado pueda juzgar con su propio criterio los aspectos y funciones de otras situaciones similares, formando hombres libres, respecto a sus juicios frente a lo nuevo.

Contextualizando las ideas, diremos que la dramatización, en el nivel escolar tiene como instrumento prioritario el diálogo, si se hace en lengua inglesa se evidencia que el estudiante procesa la información que recibe, esta acción denominada de emancipación, primero es individual y luego social y cuando desarrollan libretos con temas controversiales fortalecen su pensamiento crítico. Si bien se resalta la comunicación como consensuación de significados simbólicos, estos son dinámicos, y los nuevos significados son producto de una nueva consensuación entre los diferentes actos y es posible concretizar en la dramatización, porque el lenguaje en su relatividad es el medio de entendimiento.

Epistémicamente, la racionalidad crítica y reflexiva de un docente, se convierte en un elemento catalizador del proceso en sí, donde el estudiante inicia o adelanta su proceso de liberación que se origina en el planteamiento del hombre como problema, localiza su puesto cósmico, logra su humanización, es decir, descubre su vocación ontológica: el ser más; y su vocación histórica: la liberación (Freire, 1980). Por ello, "Utilizar el drama como recurso didáctico en la escuela es una novedosa apuesta, que viene a resultar de especial atractivo para el estudiante" (Álvarez y Martín, 2016, p.44)

Respecto al aporte psicológico, Fromm (1970) hace referencia a dos formas de aprender: en el modo tener y en el modo ser. Para esta investigación se tomó el modo ser: El estudiante recuerda activamente las palabras, las ideas, las escenas; asocia un dato sencillo que recuerda con otros, en procesos vitales. Un concepto se relaciona con otro mediante un acto productivo de pensar (o sentir) cuando se busca la palabra exacta. En esta búsqueda de lo exacto, que en Fromm es la verdad, el estudiante relaciona los conocimientos adquiridos en el aula con los objetos o procesos que forman parte de su entorno, pero no llega a expresarlos; de ser dramatizados en clase, se concretizan en dinámicas que adquieren el carácter de real, integrando ambos en un solo enunciado y logrando aprendizajes significativos.

Desde la lingǘstica, Larsen y Long (1994) y Baker (1993) explican la funcionalidad del modelo de monitor de Krashen; que, para adquirir una segunda lengua, es necesario considerar cinco principios: adquisición y aprendizaje; orden natural, monitor, el imput y el filtro afectivo. En las actividades de enseñanza-aprendizaje el monitor es la comunicación interpersonal real. La dramatización exige del desarrollo de actividades cooperativas permanentes, situación que promueve procesos activos en base al diálogo horizontal entre pares y con el docente. Concuerdan Richards y Rogers (1998) que la lengua funciona como un vehículo para el desarrollo de las relaciones personales y 
sociales, siendo el hombre por naturaleza un ser que vive y se desarrolla en sociedad. Estas teorías sirven de fundamento para validar la metodología desarrollada.

Método de enseñanza. El aprendizaje de la expresión oral del inglés fue facilitado por los pasos de la dramatización como un recurso didáctico, que consistió en un proceso de cuatro fases: a) preparación previa (conocimiento previo), b) creación de situaciones (conocimiento declarativo), c) representación (conocimiento procedimental) y d) evaluación (valoración de logros y dificultades). Estos procedimientos fueron adaptados a partir de las experiencias de Motos y Tejedo (1999) y de Tejerina (1994).

\section{METODOLOGÍA}

En la presente investigación se adoptó un diseño cuasi-experimental con pre y posprueba, donde el grupo experimental, (17 estudiantes) recibió la instrucción de la aplicación del recurso didáctico denominado dramatización, mientras que al grupo control (18) se trasfirió los contenidos mediante el método tradicional, establecido en la programación curricular. En el desarrollo de la experiencia se esperó que la mediación diseñada, tuviera un impacto positivo en la mejora del aprendizaje de la expresión oral del inglés, a diferencia del grupo control.

La población muestreada estuvo constituida por 35 estudiantes matriculados en el año académico 2019 de las secciones "A" y "B" del primer año de educación secundaria, turno matutino, cuyas edades fluctuaban entre 11 y 12 años. Los instrumentos que se utilizaron fueron una ficha de observación estructurada para valorar la eficacia de la dramatización y una lista de chequeo para medir el nivel de aprendizaje de la expresión oral del idioma inglés, constituido por 20 afirmaciones de opción múltiple tipo Likert, distribuidos en cuatro dimensiones: pronunciación (1-5); entonación (6-10); fluidez (1115) y coherencia (16-20). Para la estratificación se utilizó la escala de calificación de los aprendizajes (MINEDU,2016): Logro destacado (20-18), logro previsto (14-17), en proceso (11-13) y en inicio (0-10).

La lista de chequeo, fue elaborada en base a 20 preguntas, proporcional al número de rasgos a observar. El grado de validez fue de 88,1 ubicándolo en la condición de muy buena. La confiabilidad de consistencia interna, fue determinada con la prueba piloto Alfa de Cronbach, cuyo resultado fue 0,923 lo que indica precisión en la medición.

\section{EVALUACIÓN}

El nivel de expresión oral en el idioma inglés se evaluó en base a las dimensiones e indicadores explicitadas en el siguiente cuadro, mediante la escala de calificación de los aprendizajes en EBR, nivel secundario (MINEDU, 2016) 


\begin{tabular}{|c|c|c|}
\hline DIMENSIONES & INDICADORES & VALORES \\
\hline \multirow{6}{*}{ Pronunciación } & Consideración de las pausas. & \\
\hline & Vocalización comprensible de las palabras, frases y & Logro destacado (20-18) \\
\hline & oraciones. & Logro previsto (14-17), \\
\hline & Articulación precisa de los enunciados. & En proceso $(11-13)$ \\
\hline & Precisión en sus expresiones orales. & En inicio $(0-10)$ \\
\hline & Mediación adecuada de la expresión oral. & \\
\hline \multirow[b]{3}{*}{ Entonación } & Variación en el ritmo (velocidad y pausas). & \\
\hline & Intensidad de la expresión de sus ideas. & \\
\hline & $\begin{array}{l}\text { Volumen aceptable de la voz. Producción adecuada de } \\
\text { inflexiones tonales (acento). Expresión correcta de } \\
\text { matices semánticos. }\end{array}$ & \\
\hline \multirow{5}{*}{ Fluidez } & $\begin{array}{l}\text { Comunicación fluida (trasmisión de ideas, sentimientos } \\
\text { y experiencias). }\end{array}$ & \\
\hline & Articulación prosódica adecuada. & \\
\hline & Prolongación discursiva apropiada. & \\
\hline & Expresión espontánea de experiencias y sentimientos. & \\
\hline & ideas. & \\
\hline \multirow{5}{*}{ Coherencia } & Conexión oportuna de las ideas. & \\
\hline & Relación adecuada y continua de las ideas. & \\
\hline & Transmisión clara del mensaje. & \\
\hline & Relación semántica adecuada de las palabras. & \\
\hline & Expresión lógica del discurso. & \\
\hline
\end{tabular}

El tratamiento estadístico fue a nivel descriptivo e inferencial, el primero se realizó con la ayuda del excel y el programa estadístico SPSS versión 24. Los datos fueron procesados y presentados en tablas estadísticas de distribución absoluta y relativa simple porcentual. Para el análisis inferencial, se recurrió al cálculo e interpretación del Coeficiente de Correlación U de Mann-Whitney al 95\% de nivel de confianza, debido al carácter ordinal de la variable y la distribución no normal de los datos recolectados.

\section{RESULTADOS}

\section{Tabla 1}

Cantidad de estudiantes según el nivel de logro alcanzado en la expresión oral en el idioma inglés.

\begin{tabular}{lllllllll}
\hline & \multicolumn{4}{c}{ PRE PRUEBA } & \multicolumn{3}{c}{ POS PRUEBA } \\
\cline { 2 - 8 } $\begin{array}{l}\text { Nivel de } \\
\text { logro }\end{array}$ & \multicolumn{1}{c}{ CONTROL } & \multicolumn{2}{c}{ EXPERIMENTAL } & CONTROL & \multicolumn{2}{c}{ EXPERIMENTAL } \\
\cline { 2 - 8 } & $\mathbf{f}$ & $\mathbf{f} \%$ & $\mathbf{f}$ & $\mathbf{f} \%$ & $\mathbf{f}$ & $\mathbf{f} \%$ & $\mathbf{f}$ & $\mathbf{f} \%$ \\
\hline Inicio & 0 & $0,0 \%$ & 0 & $0,0 \%$ & 0 & $0,0 \%$ & 0 & $0,0 \%$ \\
Proceso & 16 & $88,9 \%$ & 14 & $82,4 \%$ & 14 & $77,8 \%$ & 0 & $0,0 \%$ \\
$\begin{array}{l}\text { Logro } \\
\text { previsto }\end{array}$ & 2 & $11,1 \%$ & 3 & $17,6 \%$ & 4 & $22,2 \%$ & 17 & $100,0 \%$ \\
$\begin{array}{l}\text { Logro } \\
\text { destacado }\end{array}$ & 0 & $0,0 \%$ & 0 & $0,0 \%$ & 0 & $0,0 \%$ & 0 & $0,0 \%$ \\
\hline
\end{tabular}




\begin{tabular}{lllllllll}
\hline Total & 18 & $100,0 \%$ & 17 & $100,0 \%$ & 18 & $100,0 \%$ & 17 & $100,0 \%$ \\
\hline
\end{tabular}

Nota. f: Cantidad de estudiantes. $\mathrm{f} \%$ : Porcentaje de estudiantes. Fuente: Lista de chequeo.

En la primera inferencia sobre la experiencia realizada, el impacto de la dramatización en los niveles de aprendizaje del idioma inglés es alto, tal como observamos en los resultados cuantitativos, demostrando que las escenas desarrolladas con fines académicos, estaban expresadas en el idioma inglés; ello fue un estímulo para que esos textos sean traducidos, relacionando su significado y asociando con elementos de su vida cotidiana; creando así, un aprendizaje significativo, que les servirá para los siguientes años de estudio.

\section{Tabla 2}

Cantidad de estudiantes según el nivel de logro alcanzado en la expresión oral en el idioma inglés, dimensión pronunciación.

\begin{tabular}{lllllllll}
\hline & \multicolumn{7}{c}{ PRE PRUEBA } & \multicolumn{7}{c}{ POS PRUEBA } \\
\cline { 2 - 8 } Nivel de logro & \multicolumn{2}{l}{ CONTROL } & \multicolumn{2}{c}{ EXPERIMENTAL } & CONTROL & \multicolumn{2}{c}{ EXPERIMENTAL } \\
\cline { 2 - 9 } & $\mathbf{F}$ & $\mathbf{f} \%$ & $\mathbf{f}$ & $\mathbf{f} \%$ & $\mathbf{f}$ & $\mathbf{f} \%$ & $\mathbf{f}$ & $\mathbf{f} \%$ \\
\hline Inicio & 0 & $0,0 \%$ & 0 & $0,0 \%$ & 0 & $0,0 \%$ & 0 & $0,0 \%$ \\
Proceso & 14 & $82,4 \%$ & 15 & $83,3 \%$ & 14 & $77,8 \%$ & 0 & $0,0 \%$ \\
Logro previsto & 3 & $17,6 \%$ & 3 & $16,7 \%$ & 4 & $22,2 \%$ & 17 & $100,0 \%$ \\
Logro destacado & 0 & $0,0 \%$ & 0 & $0,0 \%$ & 0 & $0,0 \%$ & 0 & $0,0 \%$ \\
\hline Total & 17 & $100,0 \%$ & 18 & $100,0 \%$ & 18 & $100,0 \%$ & 17 & $100,0 \%$ \\
\hline
\end{tabular}

Nota. f: Cantidad de estudiantes. $\mathrm{f} \%$ : Porcentaje de estudiantes. Fuente: Lista de chequeo.

La pronunciación de un idioma, diferente al materno, es siempre la tarea más difícil, los sonidos de cada fonema varían según cada idioma y para aprehender otro, hay que interiorizar la modulación. Por eso, es significativo que el 100\% de los estudiantes hayan logrado un buen nivel de pronunciación, a través de procesos de enseñanza-aprendizaje dramatizadas.

\section{Tabla 3}

Cantidad de estudiantes según el nivel de logro alcanzado en la expresión oral en el idioma inglés, dimensión Entonación.

\begin{tabular}{|c|c|c|c|c|c|c|c|c|}
\hline \multirow{3}{*}{ Nivel de logro } & \multicolumn{4}{|c|}{ PRE PRUEBA } & \multicolumn{4}{|c|}{ POS PRUEBA } \\
\hline & \multicolumn{2}{|c|}{ CONTROL } & \multicolumn{2}{|c|}{ EXPERIMENTAL } & \multicolumn{2}{|c|}{ CONTROL } & \multicolumn{2}{|c|}{ EXPERIMENTAL } \\
\hline & $\mathbf{F}$ & $\mathrm{f} \%$ & $\mathrm{f}$ & $\mathrm{f} \%$ & $\mathrm{f}$ & $\mathbf{f} \%$ & $\mathrm{f}$ & $\mathbf{f} \%$ \\
\hline Inicio & 0 & $0,0 \%$ & 0 & $0,0 \%$ & 0 & $0,0 \%$ & 0 & $0,0 \%$ \\
\hline Proceso & 15 & $83,3 \%$ & 14 & $82,4 \%$ & 14 & $77,8 \%$ & 2 & $11,8 \%$ \\
\hline Logro previsto & 3 & $16,7 \%$ & 3 & $17,6 \%$ & 4 & $22,2 \%$ & 15 & $88,2 \%$ \\
\hline Logro destacado & 0 & $0,0 \%$ & 0 & $0,0 \%$ & 0 & $0,0 \%$ & 0 & $0,0 \%$ \\
\hline Total & 18 & $100,0 \%$ & 17 & $100,0 \%$ & 18 & $100,0 \%$ & 17 & $100,0 \%$ \\
\hline
\end{tabular}

Nota. f: Cantidad de estudiantes. $\mathrm{f} \%$ : Porcentaje de estudiantes. Fuente: Lista de chequeo. 
La entonación de las palabras, en cualquier idioma, es la que les da el valor de uso, por lo tanto, sus significados y significantes están relacionados a la entonación que se da a las palabras, frases y oraciones de uso frecuente. En ese contexto, es alentador el aumento del número de estudiantes $(88,2 \%)$ que alcanzaron un nivel de logro previsto.

\section{Tabla 4}

Cantidad de estudiantes según el nivel de logro alcanzado en la expresión oral en el idioma inglés, dimensión fluider:

\begin{tabular}{lllllllll}
\hline & \multicolumn{7}{c}{ PRE PRUEBA } & \multicolumn{5}{c}{ POS PRUEBA } \\
\cline { 2 - 9 } Nivel de logro & CONTROL & \multicolumn{2}{l}{ EXPERIMENTAL } & CONTROL & \multicolumn{2}{c}{ EXPERIMENTAL } \\
\cline { 2 - 9 } & $\mathbf{F}$ & $\mathbf{f} \%$ & $\mathbf{f}$ & $\mathbf{f} \%$ & $\mathbf{f}$ & $\mathbf{f} \%$ & $\mathbf{f}$ & $\mathbf{f} \%$ \\
\hline Inicio & 1 & $5,6 \%$ & 0 & $0,0 \%$ & 0 & $0,0 \%$ & 0 & $0,0 \%$ \\
Proceso & 17 & $94,4 \%$ & 17 & $100,0 \%$ & 15 & $83,3 \%$ & 1 & $5,9 \%$ \\
Logro previsto & 0 & $0,0 \%$ & 0 & $0,0 \%$ & 3 & $16,7 \%$ & 16 & $94,1 \%$ \\
Logro destacado & 0 & $0,0 \%$ & 0 & $0,0 \%$ & 0 & $0,0 \%$ & 0 & $0,0 \%$ \\
\hline Total & 18 & $100,0 \%$ & 17 & $100,0 \%$ & 18 & $100,0 \%$ & 17 & $100,0 \%$ \\
\hline
\end{tabular}

Nota. f: Cantidad de estudiantes. $\mathrm{f} \%$ : Porcentaje de estudiantes. Fuente: Lista de chequeo.

La fluidez en el uso de un idioma, es el producto del dominio de las otras dimensiones, por ello, es alentador el resultado obtenido (94,1\%) lo que demuestra, que el uso adecuado de la dramatización, es altamente eficaz, en el aprendizaje de una lengua extranjera. Por otro lado, consideramos que la fluidez, es un medio no natural, pero lograron mejorar su nivel mediada por las presentaciones, se espera que pueda ser útil en la cotidianidad del estudiante.

\section{Tabla 5}

Cantidad de estudiantes según el nivel de logro alcanzado en la expresión oral en el idioma inglés, dimensión coherencia.

\begin{tabular}{lllllllll}
\hline & \multicolumn{7}{c}{ PRE PRUEBA } & \multicolumn{5}{c}{ POS PRUEBA } \\
\cline { 2 - 9 } Nivel de logro & \multicolumn{1}{l}{ CONTROL } & \multicolumn{2}{l}{ EXPERIMENTAL } & \multicolumn{1}{l}{ CONTROL } & \multicolumn{2}{c}{ EXPERIMENTAL } \\
\cline { 2 - 9 } & $\mathbf{F}$ & $\mathbf{f} \%$ & $\mathbf{f}$ & $\mathbf{f} \%$ & $\mathbf{f}$ & $\mathbf{f} \%$ & $\mathbf{f}$ & $\mathbf{f} \%$ \\
\hline Inicio & 0 & $0,0 \%$ & 1 & $5,9 \%$ & 0 & $0,0 \%$ & 0 & $0,0 \%$ \\
Proceso & 15 & $83,3 \%$ & 15 & $88,2 \%$ & 14 & $77,8 \%$ & 2 & $11,8 \%$ \\
Logro previsto & 3 & $16,7 \%$ & 1 & $5,9 \%$ & 4 & $22,2 \%$ & 15 & $88,2 \%$ \\
Logro destacado & 0 & $0,0 \%$ & 0 & $0,0 \%$ & 0 & $0,0 \%$ & 0 & $0,0 \%$ \\
\hline Total & 18 & $100,0 \%$ & 17 & $100,0 \%$ & 18 & $100,0 \%$ & 17 & $100,0 \%$ \\
\hline
\end{tabular}

Nota. f: Cantidad de estudiantes. $\mathrm{f} \%$ : Porcentaje de estudiantes. Fuente: Lista de chequeo.

La coherencia en el empleo de palabras de un idioma extranjero, es muy difícil de alcanzar, porque el hablante no piensa en ese idioma; por eso es sorprendente la eficacia del método basado en la dramatización para mejorar el aprendizaje oral del idioma inglés, los altos niveles de logro $(88,2 \%)$, debe ser resaltado, en busca de su generalización. 


\section{DISCUSIÓN}

En términos generales, los resultados obtenidos cuantitativa y cualitativamente concuerdan con los logros de Salas (2017) quien implementó técnicas teatrales mediante la creación de guiones en la lengua inglesa y evidencia que en el grupo de undécimo año, la totalidad de la población logró mejorar aspectos de pronunciación, gramática, vocabulario y fluidez. En el caso, del grupo de séptimo, el 86,9\% logró los objetivos de la asignatura, resultados que corroboran el nivel obtenido en la dimensión fluidez verbal, donde el 94,1\% de estudiantes alcanzaron ubicarse en el nivel de logro previsto.

Para López y otros (2009) la dramatización es el arte de la palabra, de la expresión desde el prisma del género dramático que permite la exploración del universo cultural al que paulatinamente se adentran el estudiante. Heldebrand (2003) en su investigación concluye, que el drama propicia una forma relajada e informal de aprendizaje; por ello, ayuda a la pronunciación y entonación de la terminología inglesa. Aspectos que concuerdan con los resultados de esta investigación (100\% en pronunciación y 88,2\% en entonación). Gonzales (1997) coincide al manifestar que las ideas que se expresan deben tener secuencia lógica, siempre y cuando el alumno sea capaz de interpretar el texto. Consecuentemente, la dramatización crea una atmósfera de seguridad para la mejor comprensión de la cultura. No menos importante son los resultados obtenidos por Torres (2017) quien afirma que una segunda lengua se aprende de manera natural, en un ambiente adecuado, para que los estudiantes puedan hacer uso del vocabulario y de la estructura gramatical como una necesidad cotidiana, para ello, el drama es un mecanismo eficaz. Para Gudiño (2017) este recurso didáctico contribuye a fortalecer las habilidades de comunicación oral, escrita y corporal de acuerdo a las necesidades de cada estudiante. Efectivamente, en nuestro caso, el resultado lo evidencia ya que el 100\% de los estudiantes lograron ubicarse en un nivel significativo de aprendizaje de la expresión oral del inglés.

\section{CONCLUSIONES}

- Son suficientes las evidencias empíricas que indican que la aplicación de la dramatización como recurso didáctico influye significativamente en el nivel de desarrollo de la expresión oral en el idioma inglés, es decir que el 92,6\% de los estudiantes consiguieron ubicarse en logro previsto, la interiorización de un personaje y el drama que viven en el escenario, hacen que el aprendizaje de un segundo idioma sea más sencillo, asociado con los procesos que representa, mejor si el estudiante se identifica con el representado.

- Las experiencias desarrolladas revelan que la aplicación adecuada de la dramatización, mejora el nivel de desarrollo de la entonación y pronunciación del idioma inglés, lo que indica que, los estudiantes aprendieron a darle el valor de uso, por lo tanto, sus significados y significantes están relacionados a la variación del tono de la voz. En cuanto a la fluidez de la expresión, los resultados son satisfactorios, la dramatización es un medio no natural, pues las voces se impostan, los cuerpos se adecuan a la exigencia de un guion, y proyecta sus resultados a la vida cotidiana del estudiante.

- Finalmente, la coherencia de las ideas, en muchos de los casos no es lógica, sin embargo, las actividades de la dramatización mostraron su efectividad, ya que los 
datos cuantitativos que se obtuvo son alentadores. La eficacia del recurso didáctico estudiado es sorprendente, razón suficiente para ser incorporado en las acciones educativas de las sesiones de aprendizaje del idioma inglés y sus logros deben ser generalizados.

\section{REFERENCIAS BIBLIOGRÁFICAS}

Álvarez, P. y Martín, A. (2016). El teatro como herramienta didáctica en la enseñanza de la historia de la educación contemporánea. Revista digital de investigación en docencia universitaria.10(1), pp. 41-51. https://doi.org/10.19083/ridu.10.459

Arksey, H. y O’Malley, L. (2005). Scoping studies: towards a methodological framework. International Journal of Social Research Methodology, (8)1, pp.19-32, DOI: 10.1080/1364557032000119616

Baker, C. (1993). Fundamentos de educación bilingüe y bilingüismo. Cátedra

Boquete, M. (2011). El uso del juego dramático en la enseñanza de lenguas: las destrezas orales. [Tesis doctoral, Universidad de Alcalá] Repositorio institucional https://ebuah.uah.es/dspace/handle/10017/16981

Cervera, J. (1992). Dramatización y teatro: precisiones terminológicas y conceptuales. Universidad de Valencia. https://core.ac.uk/download/pdf/61902078.pdf

Chávez, M. X.; Saltos, M. A. y Saltos, C. M. (2017). La importancia del aprendizaje y conocimiento del idioma inglés en la enseñanza superior. Revista Científica Dominio de las Ciencias. 3(3), pp. 759-771. https://dialnet.unirioja.es/servlet/ articulo? codigo $=6234740$

Chiclote, C. (2005). Uso de actividades lúdicas para mejorar la pronunciación de los fonemas vocálicos 111 y /i: 1 en los alumnos de tercer grado de educación secundaria [Tesis de maestría] Universidad de Lambayeque.

Dirección Regional de Educación Huancavelica-DREH (2017). Reporte del informe del concurso 2017.

Desialova, L. (2009). Using different forms of Drama in EFL. Classroom. Humanising LanguageTeaching. http://www.hltmag.co.uk/aug09/sart07.htm

English First (2016). EF Education First. EF EPI: EF English proficiency index. http://www.ef.edu/epi/downloads/.

España, C. (2009). El idioma inglés en el currículo universitario: importancia, retos y alcances. Revista Electrónica Educare. 14(2), pp. 63-69, ISSN: 1409-42-58. https://www.redalyc.org/pdf/1941/194115606005.pdf

Fromm, E. (1970). Tener y ser. https://jesuitas.lat/uploads/tener-y-ser/ERICH\%20 FROMM\%20-\%201976\%20-\%20 TENER\%20Y\%20SER.pdf

Fuegel, C. (2000). Innovemos el aula: creatividad, grupos y dramatización. Octaedro.

Gonzáles, T. (1997). Pruebas de dominio lector para alumnos de enseñanza básica. Ediciones Universidad Católica de Chile. 
Gudiño, E. (2017). La Dramatización en el desarrollo de la producción oral del idioma inglés. (Tesis de Licenciatura) Universidad Central del Ecuador. http://www.dspace.uce.edu.ec/bitstream/25000/13662/1/T-UCE-0010-0102017.pdf

Habermas, J. (2007). Teoría de la acción comunicativa I: Racionalidad social y racionalización de la acción. TAURUS.

Heldenbrand, B. (2003). Drama techniques in English language learning. The Korea Tesol Journal, 6 (1), pp. 27-38. https://koreatesol.org/sites/default/files/pdf_ publications/KTJ6-2003web.pdf

Instituto de Integración (2016). Informe. http://www.integracion.pe/28-alumnoscolegios-publicos-estudian-mas-horas-al-dia/.

Internet world stats (2017). Idiomas en la red. https://es.statista.com/grafico/7736/lamitad-del-contenido-de-internet-esta-en-ingles/

Larsen, D. y Long, F. (1994). Introducción a la adquisición de segundas lenguas. Madrid: Grados.

Llanos, M. (2018). Niveles de aprendizaje, en los resultados de evaluación censal de los estudiantes 2015, en la IEs "Juan Velazco Alvarado" Totoroma. [Tesis de 2da. Especialidad, Universidad San Ignacio de Loyola] Repositorio institucional http://repositorio.usil.edu.pe/bitstream/USIL/8075/4/2018_LLANOS_HUAR AHUARA_MANUEL.pdf

López, A.; Jerez, I. y Encabo, E. (2009). Claves para una enseñanza artístico creativa. Dramatización. Octaedro.

Ministerio de Educación (2016). Plan de implementación al 2021 de la política nacional de enseñanza, aprendizaje y uso de idioma inglés - Política "Inglés, puertas al mundo." http:// www.minedu.gob.pe/ingles-puertas-al-mundo/.

Motos, T. y Tejedo, F. (1999). Prácticas de dramatización. La Avispa.

Ninamango, P. (2008). Actitudes hacia el aprendizaje del idioma inglés en estudiantes de educación secundaria de Gestión Pública en el Perú. [Tesis de maestría, Universidad Nacional de Educación.] Repositorio institucional http://repositorio.une.edu.pe/ bitstream/handle/UNE/1124/TL\%20SH-Lxii\%20I69\%202014.pdf?sequence= 1\&isAllowed $=\mathrm{y}$

Pérez, I. (2015). La importancia del idioma inglés. [Artículo] Fundación Luis Amigo. https://www.researchgate.net/publication/281915812_La_importancia_del_idio ma_ingles

Reyes, D. y Echevarría, M. (2016). La pronunciación y la enseñanza de inglés en las Ciencias Médicas. Revista Habanera de Ciencias Médicas. (15)2, pp. 1-8 http://scielo.sld.cu/scielo.php?script=sci_arttext\&pid=S1729-519X20160002000 14

Richards, J. y Rogers, T. (1998). Enfoques y métodos en la enseñanza del idioma. University Press. 
Salas, J. (2017). El uso del teatro en la clase de inglés como estrategia didáctica. Revista Inter Sedes. 17 (36), pp.1-56 San José. Universidad de Costa Rica. http://dx.doi.org/10.15517/isucr.v17i36.27467

Tejerina, I. (1993). Dramatización y teatro infantil. Siglo XXI

Torres, A. (2017). El drama como herramienta en el aula de lengua inglesa. (Tesis de grado) Universidad de Sevilla. https://idus.us.es/bitstream/handle/11441/63009/ TFG\%20ADORACION\%20TORRES\%20PARISH.pdf?sequence=1

UNESCO (2003). La educación en un mundo plurilingüe. [Documento de orientación] Organización de las Naciones Unidas para la Educación, la Ciencia y la Cultura. https://www. corteidh.or.cr/tablas/25016.pdf

Vygotsky, I. (1995). El desarrollo de los procesos psicológicos superiores. Grijalbo.

\section{CITAR COMO:}

Hermoza Palomino, W. (2021). Dramatización como recurso didáctico y expresión oral del inglés en estudiantes de educación secundaria. Puriq, 3(2), 253-265. https://doi.org/10.37073/puriq.3.2.156 